\title{
Produção e consumo de energia elétrica fotovoltaica: um aplicativo destinado ao ensino de Física e ao debate sobre a matriz energética
}

\author{
Production and consumption of photovoltaic electricity: an application for teaching Physics and
}

debate the energy matrix

Producción y consumo de electricidad fotovoltaica: una aplicación para la enseñanza de la Física y

el debate sobre la matriz energética

Recebido: 07/11/2021 | Revisado: 13/11/2021 | Aceito: 16/11/2021 | Publicado: 26/11/2021

\author{
Antônio Carlos da Cruz Junior \\ ORCID: https://orcid.org/0000-0003-0415-1191 \\ Universidade Federal de Alfenas, Brasil \\ E-mail: antonio.cruz@sou.unifal-mg.edu.br \\ Samuel Bueno Soltau \\ ORCID: https://orcid.org/0000-0002-7211-2533 \\ Universidade Federal de Alfenas, Brasil \\ E-mail: samuel.soltau@unifal-mg.edu.br
}

\begin{abstract}
Resumo
A busca por fontes de energia alternativa, renovável, limpa e sustentável é premente na civilização contemporânea e área de intensa pesquisa científica e tecnológica. O ensino das Ciências, em particular da Física, é instado a contribuir através da oferta de subsídios teóricos e conceituais aos alunos, a fim de que possam entender, refletir e participar do debate a respeito do tema como cidadãos. Como contribuição para esta demanda de ensino desenvolveu-se, com propósitos didáticos, um aplicativo para dispositivos móveis que simula o funcionamento de um sistema de energia solar fotovoltaico baseado efetivamente em parâmetros técnicos do estado da arte atual. Como resultado obteve-se um aplicativo com visual simples, flexível e intuitivo, capaz de atender exigências didáticas de aulas remotas e presenciais em qualquer série do Ensino Médio. A criação do aplicativo alinha-se às tendências tecnológicas contemporâneas de ubiquidade e mobilidade dos recursos potenciais de aprendizagem na sociedade, intensamente conectada por tecnologias digitais de comunicação e se constitui numa alternativa viável para o ensino dos temas relacionados ao uso da energia solar fotovoltaica capaz de contribuir para o ensino e as reflexões a respeito da matriz energética contemporânea.

Palavras-chave: Energias renováveis; Eletricidade fotovoltaica solar; Aplicativo para dispositivos móveis; Ensino de Física.

Abstract

The search for alternative, renewable, clean and sustainable energy sources is urgent in contemporary civilization and an area of intense scientific and technological research. The teaching of Science, in particular Physics, is urged to contribute by offering theoretical and conceptual subsidies to students, so that they can understand, reflect and participate in the debate on the subject as citizens. As a contribution to this educational demand, a mobile device application was developed for didactic purposes that simulates the operation of a photovoltaic solar energy system based effectively on technical parameters of the current state of the art. As a result, we obtained an application with a simple, flexible and intuitive interface, capable of addressing the didactic requirements of remote and face-to-face classes in any high school grade. The creation of the application is aligned with contemporary technological trends of ubiquity and mobility of potential learning resources in society, intensely connected by digital communication technologies and constitutes a viable alternative for teaching topics related to the use of photovoltaic solar energy capable of contribute to teaching and reflections about the contemporary energy matrix.
\end{abstract}

Keywords: Renewable energy; Solar photovoltaic electricity; Mobile app; Physics teaching.

\section{Resumen}

La búsqueda de fuentes de energía alternativas, renovables, limpias y sostenibles es una cuestión acuciante en la civilización contemporánea y un ámbito de intensa investigación científica y tecnológica. Se insta a la enseñanza de las Ciencias, en particular de la Física, a contribuir ofreciendo apoyo teórico y conceptual a los estudiantes, para que comprendan, reflexionen y participen en el debate sobre el tema como ciudadanos. Como aporte a esta demanda educativa, se desarrolló una aplicación para dispositivos móviles, con fines didácticos, que simula el funcionamiento de un sistema de energía solar fotovoltaica basándose efectivamente en parámetros del estado de la técnica actual. Como resultado, obtuvimos una aplicación con un aspecto sencilla, flexible e intuitivo, capaz de cumplir con los requisitos 
didácticos de las clases presenciales y remotas en cualquier grado de secundaria. La creación de la aplicación se alinea con las tendencias tecnológicas contemporáneas de ubicuidad y movilidad de los recursos potenciales de aprendizaje en la sociedad, intensamente conectados por las tecnologías de la comunicación digital y constituye una alternativa viable para la enseñanza de temas relacionados con el uso de la energía solar fotovoltaica capaz de contribuir a la docencia y reflexiones sobre la matriz energética contemporánea.

Palabras clave: Energías renovables; Electricidad solar fotovoltaica; Aplicación para dispositivos móviles; Enseñanza de la Física.

\section{Introdução}

A radiação solar é a principal contribuição energética que viabiliza os mais variados processos físicos, químicos e biológicos, com destaque para a existência e o suporte de vida no planeta Terra. Quando a radiação solar interage com o sistema atmosférico da Terra, uma parte é refletida de volta para o espaço e outra parte é absorvida pela superfície do planeta. A maioria da energia do Sol é emitida a partir de sua superfície, onde a temperatura é de aproximadamente $5727^{\circ} \mathrm{C}$, enquanto a temperatura média da superfície da Terra é cerca de $15^{\circ} \mathrm{C}$ (Wendisch \&Yang, 2012).

A captação do calor e da luz solar e a sua conversão em outras formas de energia para a realização de trabalho útil por meio de tecnologias é considerada na atualidade uma fonte de energia alternativa, renovável, limpa e sustentável do ponto de vista econômico e ecológico (Sobczyk et al., 2017; Corrêa \& Benite, 2020). A captação de energia solar para a produção de energia elétrica por tecnologias baseadas no efeito fotovoltaico é uma das alternativas mais viáveis na atualidade, cabendo estudar as especificidades de cada localidade (Maimoni \& Cardoso, 2020). As células solares são usadas em uma variedade de aplicações, desde a alimentação de relógios e calculadoras até o carregamento de baterias para barcos e sistemas de comunicação e o meio de fornecer energia para a maioria dos satélites artificiais lançados desde o início dos programas espaciais (Duffie, Beckman \& Blair, 2020). Porém, cabe ressaltar que há outros tipos de aproveitamento da energia solar, tais como a energia termossolar e a energia heliotérmica, isso sem considerar que a energia eólica é, também, indiretamente decorrente da energia solar (Silva, 2010).

O estágio atual do conhecimento em Física descreve a radiação eletromagnética como tendo um comportamento tanto corpuscular, discreto, quanto ondulatório e contínuo (Griffiths, 2017). O estudo da captação, armazenamento e consumo da energia solar para a geração de energia elétrica envolve ambos os comportamentos conforme a escala de análise e o meio de observação, o que qualifica seu estudo como um tema privilegiado que perpassa diversas áreas da Física e, portanto, viabiliza a sua abordagem em diversos níveis do ensino da Física, como corroboram os trabalhos de Silva et al. (2017), Lima (2018) e Rocha e Costa (2019).

Este artigo apresenta e descreve um aplicativo criado para smartphones e tablets desenvolvido com fins didáticos que simula o funcionamento de um sistema de captação, armazenamento e consumo de energia solar fotovoltaico do tipo off-grid. O aplicativo se oferece como um subsídio para ser integrado tanto a propostas de ensino em sala de aula quanto às fundamentadas em aprendizagem ubíqua (Hightower \& Borrirello, 2001; Santaella, 2013) que pretendam explorar o potencial inerente ao tema e sua capacidade para desencadear diversas discussões tanto no âmbito da Física quanto de outras disciplinas escolares cujo tema da energia seja pertinente.

A motivação inicial da proposta foi a de apresentar aos alunos do Ensino Médio os conceitos físicos essenciais relacionados ao tema das fontes de energia alternativa, renovável, limpa e sustentável, em particular as que já estão atualmente em plenas condições tecnológicas de uso prático, com custos acessíveis ao cidadão comum e não apenas às indústrias e empresas de grande porte. Excluíram-se da lista, portanto, todas as alternativas que estão em fase experimental ou são incipientes demais para ter uso prático efetivo, aquelas cujos custos são proibitivos. Ao aplicar estas restrições emergiu como favorita para o propósito didático a energia fotovoltaica solar.

Em termos de organização, na primeira parte deste artigo elenca-se e aborda-se brevemente a dinâmica de fenômenos energéticos envolvendo o sistema Sol-Terra, percorre os conceitos físicos pertinentes para o entendimento da captação de energia 
solar e sua conversão em energia elétrica útil. Em seguida, descreve-se os tipos de sistemas atualmente existentes para a transformação de energia solar em elétrica e as suas características essenciais, concentrando-se nos aspectos que foram contemplados na modelagem contida no aplicativo desenvolvido. A próxima seção apresenta, descreve as funcionalidades do aplicativo desenvolvido e discute alguns usos didáticos potenciais com o uso do aplicativo. E, finalizando apresentamos as considerações finais e algumas melhorias que serão incluídas em breve na próxima versão do aplicativo.

\section{Aspectos Físicos Pertinentes e Conceitos Relevantes}

Esta breve revisão não tem a pretensão de esgotar ou aprofundar os conceitos físicos envolvidos no uso da energia solar para gerar energia elétrica, mas apenas oferecer um panorama geral que permita aferir o quão vasto é o assunto e o potencial didático que pode ser explorado, em particular no ensino médio com o uso do aplicativo desenvolvido.

O conceito central e mais fundamental para iniciar a entender a viabilidade do processo de produção para consumo em escala domiciliar e até industrial de fotoeletricidade é o de balanço de radiação que será definido aqui, por simplicidade, apenas como a diferença entre a entrada e a saída de energia de um sistema físico (Horton et al., 1996). Considerando a interação energética entre a Terra e o Sol, pode-se identificar a superfície terrestre, a atmosfera e as nuvens como os componentes mais básicos para compreender o balanço de radiação no sistema solar-terrestre.

Ao ingressar no sistema atmosférico terrestre a radiação solar é parcialmente absorvida pela superfície do planeta. Outra parcela é refletida e retorna ao espaço. A maior parte da energia do Sol é emitida a partir de sua superfície, onde a temperatura alcança aproximadamente $5727^{\circ} \mathrm{C}$. A temperatura média da superfície da Terra é de $15^{\circ} \mathrm{C}$, ou seja, o Sol irradia muito mais energia que a Terra. Sabe-se que grande parte da radiação solar se situa na faixa espectral ao redor de $0,5 \mathrm{~m}$, ou seja, trata-se de uma radiação de ondas curtas, enquanto a radiação terrestre por estar na faixa de $10 \mathrm{~m}$ é uma radiação de ondas longas (Von Schuckmann et al., 2020).

Segundo Von Schuckmann et al. (2020), há três processos que influenciam na distribuição e, consequentemente, no balanço de radiação do sistema Terra-Sol. Tais processos são: espalhamento e reflexão, absorção atmosférica e absorção da superfície. Cerca de 30\% da radiação solar é espalhada ou refletida, cerca de 19\% é absorvida pela atmosfera terrestre e 51\% da radiação solar é então absorvida pela superfície do planeta.

Ao considerar cada processo em particular para analisar o que ocorre com cada porção de energia, os estudos revelam que ao considerar o total de energia solar perdida por espalhamento e reflexão, tem-se que aproximadamente $6 \%$ é espalhada para o espaço desde a atmosfera e cerca de $20 \%$ é refletida pela cobertura de nuvens, restando apenas cerca de $4 \%$ refletida pela superfície da Terra.

Processos de absorção atmosférica, responsáveis por cerca de 19\% do destino da radiação solar incidente é distribuída em cerca de $3 \%$ da radiação solar absorvida pelas nuvens e cerca de $16 \%$ absorvido por vapor de água, poeira e outros componentes em suspensão na atmosfera terrestre (Von Schuckmann et al., 2020).

Quanto a absorção pela superfície do solo, cerca de $25 \%$ alcança e penetra na superfície da Terra com interferência desprezível da atmosfera e, aproximadamente $26 \%$ da radiação é difundida para a superfície. A cobertura vegetal do solo, oceanos, lagos e rios têm papel preponderante no processo de absorção da radiação solar pela superfície do planeta. A vegetação absorve grande quantidade de radiação incidente impedindo assim alcançar a superfície do terreno. Parte da energia que incide na vegetação é consumida em processos bioquímicos, como a fotossíntese e na evaporação o que tem implicações no balanço de energia. A Tabela 1 reúne uma síntese dos processos atmosféricos a serem considerados no balanço de radiação anteriormente descritos. 
Tabela 1 - Síntese dos processos atmosféricos radiativos.

\begin{tabular}{cl}
\hline Espalhamento e Reflexão & $\bullet 6 \%$ é espalhada para o espaço pelos gases atmosféricos \\
$(30 \%)$ & $\bullet 20 \%$ é refletida pela presença de nuvens \\
\hline \multirow{2}{*}{$\begin{array}{c}\text { Absorção atmosférica } \\
(19 \%)\end{array}$} & $\bullet-16 \%$ é refletida pela superfície terrestre \\
\hline $\begin{array}{c}\text { Absorção da superfície } \\
(51 \%)\end{array}$ & $\bullet 26 \%$ radiação difundida para a superfície solar é absorvida pelas nuvens \\
& $-25 \%$ penetra direto na superfície terrestre sem outras interações com a atmosfera \\
\hline
\end{tabular}

Fonte: Elaborada pelos autores a partir de Von Schuckmann et al. (2020)

Considerando a importância que cada um destes processos tem no balanço de radiação e, consequentemente para o aproveitamento da energia solar, vale ressaltar a seguir alguns aspectos em particular.

\subsection{Espalhamento}

Embora a radiação solar possa ser considerada para todos os efeitos práticos como incidindo em linha reta, os gases e aerossóis causam espalhamento do feixe. A insolação, isto é, a quantidade de tempo em horas que o Sol brilha no céu num determinado dia, torna-se difusa. Deste modo a radiação solar é espalhada ou refletida causando a claridade do céu durante o dia e a iluminação de áreas não iluminadas diretamente do Sol (Lu et al., 2021).

De acordo com Lu et al. (2021), variações no espalhamento dependem do tamanho das moléculas de gás ou aerossóis com os quais interagem na atmosfera. O espalhamento por partículas que têm o raio menor do que o comprimento de onda da radiação espalhada depende do comprimento de onda. Se o raio das partículas é aproximadamente igual ou maior do que cerca de até oito vezes o comprimento de onda da radiação espalhada, o espalhamento independe do comprimento de onda e a distribuição angular da radiação espalhada pode ser descrita pelas leis da ótica geométrica.

\subsection{Reflexão}

A reflexão ocorre no limite entre dois meios refringentes diferentes, quando parte da radiação que atinge este limite retorna. A parte da radiação refletida por uma superfície é denominada albedo. Albedo é a medida da quantidade de radiação solar refletida por um corpo ou uma superfície e é calculado como a razão entre a quantidade de radiação refletida e a quantidade de radiação recebida. Em termos gerais, o albedo é a relação entre a quantidade de luz refletida pela superfície terrestre e a quantidade de luz recebida do Sol afetando diretamente a temperatura de equilíbrio da Terra. Além disso, o albedo varia no espaço e no tempo dependendo da natureza da superfície e da altura do Sol (Lu et al., 2021).

Nas camadas internas da atmosfera, os topos das nuvens são os refletores mais significativos. O albedo dos topos de nuvens depende de sua espessura, variando de menos de $40 \%$ para nuvens finas a $80 \%$ para nuvens espessas. O albedo total terrestre é cerca de 30\% segundo Von Schuckmann et al. (2020).

\subsection{Absorção na Atmosfera}

Ao absorver radiação as moléculas aumentam a vibração interna que resulta, por seu turno em aumento da sua temperatura. Por este motivo, os gases que absorvem melhor a radiação, contribuem mais para o aquecimento atmosférico. Como o vapor d'água possui alto índice de absorção de radiação solar, principalmente na faixa do infravermelho, contribui, junto com 
o oxigênio e o ozônio, para a maior parte dos cerca $19 \%$ da radiação solar que é absorvida na atmosfera, em particular na troposfera onde existe a maior concentração de vapor d'água (Nasa, 2021).

\subsection{Absorção na Superfície}

Cerca de $51 \%$ da energia solar incidente no topo da atmosfera terrestre atinge a superfície. A maior parte da energia que atinge a superfície retorna à atmosfera, irradiada de volta na faixa do infravermelho. $\mathrm{O}$ vapor d'água e o dióxido de carbono são os principais gases absorvedores da radiação infravermelha. Von Schuckmann et al. (2020) ressaltam que o vapor d'água absorve aproximadamente cinco vezes mais radiação terrestre que todos os outros gases combinados.

Como os gases atmosféricos são transparentes para as ondas curtas da radiação solar e absorventes para as ondas longas da radiação terrestre, resulta que a atmosfera é aquecida a partir da radiação emitida pela superfície da própria Terra. Tal efeito contribui em parte para o efeito estufa (Nasa, 2021).

\subsection{Balanço de Energia Solar}

Medir a incidência da radiação solar e suas componentes direta e difusa na superfície terrestre é essencial para os estudos de sua influência no clima e na atmosféricas e essencial para escolher sítios apropriados para implantar instalações de sistemas de produção de energia fotovoltaica e garantir o máximo aproveitamento de energia, considerando que há variações de intensidade da radiação solar ao longo do ano.

A radiação global pode ser estimada a partir do número de horas de insolação. Considere-se um feixe de radiação incidente, $Q_{O}$, capaz de penetrar a atmosfera e alcançar o limite extremo das camadas atmosféricas. Durante seu percurso parte da radiação incidente é absorvida, outra parcela é difundida e, finalmente, uma terceira parte atinge a superfície terrestre. A fração difundida pela atmosfera constitui uma parte que retorna ao espaço sideral e a outra parte que atinge a superfície terrestre e é denominada radiação do céu, $Q_{c}$. A parcela de radiação que não se alterou ao atravessar a atmosfera é denominada radiação direta, $Q_{d}$. Assim, a radiação que por fim chega até a superfície terrestre é denominada de radiação global, $Q_{g}$, e pode ser calculada, segundo Kejna, Uscka-Kowalkowska e Kejna (2021), pela Equação 1 cuja unidade da radiação solar é $\mathrm{cal} / \mathrm{cm}^{2} \cdot \mathrm{dia}$.

$$
Q_{g}=Q_{c}+Q_{d}
$$

Os processos que decorrem da interação da atmosfera terrestre com a radiação solar e os conceitos físicos relacionados examinados aqui são pertinentes para fornecer um panorama da dinâmica que finalmente resulta na energia disponível para ser captada e convertida em eletricidade capaz de realizar trabalho útil, seja acionando os aparelhos eletrodomésticos, máquinas e equipamentos nas indústrias (Duffie, Beckman \& Blair, 2020).

Para complementar os fenômenos naturais e os conceitos físicos associados com o uso da energia solar e a possibilidade de transformação em energia elétrica, cabe examinar, ainda que brevemente, o fenômeno mais diretamente vinculado à toda a tecnologia: efeito fotoelétrico.

\section{Efeito Fotoelétrico}

O fenômeno da fotoeletricidade foi descoberto em 1886 por Heinrich Hertz (1857-1894), porém somente explicado em 1905 por Albert Einstein (1879-1955) ao aplicar a ideia de quantização da energia introduzida em 1900 por Max Planck (18581947). O efeito fotoelétrico é o que permite em última análise a possibilidade de transformar a energia solar em eletricidade (Rablau et al., 2019). 
De modo simples, o efeito fotoelétrico é um fenômeno físico que consiste na ejeção de elétrons - que neste caso em particular são denominados fotoelétrons - por alguns materiais, em geral metais, ao serem submetidos à radiação eletromagnética de frequências específicas. Nesse fenômeno, a luz é descrita no seu aspecto corpuscular, ou quantizado, transferindo os quanta de energia - fótons - para os elétrons que são ejetados para fora da superfície do material. Vale ressaltar que os fotoelétrons somente são ejetados caso a energia dos fótons incidentes seja maior ou igual à função trabalho do material. A energia cinética dos elétrons ejetados somente depende da frequência da radiação incidente e a intensidade da radiação afeta apenas a quantidade de fotoelétrons que é ejetada a cada segundo.

Pela teoria corpuscular proposta por Planck e utilizada por Einstein para explicar o efeito fotoelétrico, a luz é formada por grande quantidade de fótons, os quanta de luz sem massa que carregam uma quantidade exata de energia que é proporcional à frequência da luz vezes a constante de Planck, como na Equação 2:

$$
E=h \cdot v
$$

onde $E$ é a energia, $h$ é a constante de Planck que vale $6,662 \times 10^{-34} \mathrm{~J} \cdot$ se a frequência da radiação, $v$.

Caso a energia de um fóton seja suficiente, pode provocar a ejeção de fotoelétrons do material. A energia cinética de um fotoelétron ejetado pode ser calculada pela Equação 3, onde a energia cinética, $K$, adquirida pelos fotoelétrons é diretamente proporcional à energia, $E$, dos fótons incidentes, bem como da função trabalho, $\Phi$.

$$
K=E-\Phi
$$

Esta equação fornece uma medida da quantidade de energia potencial pela qual os elétrons estão ligados ao núcleo atômico, ou seja, é uma medida da quantidade mínima de energia necessária para ejetá-los da superfície do material (cf. Tabela 2). A função trabalho, $\Phi$, refere-se à quantidade mínima de energia necessária para ejetar fotoelétrons de uma superfície material.

Tabela 2 - Função trabalho de alguns elementos.

\begin{tabular}{|c|c|}
\hline Elemento & Função trabalho $(\boldsymbol{\Phi} / \mathbf{e V})$ \\
\hline Alumínio & $4,06-4,26$ \\
\hline Cobre & $4,53-5,10$ \\
\hline Ferro & $4,67-4,81$ \\
\hline Ouro & $5,10-5,47$ \\
\hline Platina & $5,12-5,93$ \\
\hline Zinco & $3,63-4,90$ \\
\hline
\end{tabular}

Fonte: Workfunction Values (2020).

Note que a energia excedente é transferida para os fotoelétrons na forma de energia cinética. Ressalte-se que a energia cinética adquirida pelos fotoelétrons depende de modo único e exclusivo da frequência da radiação eletromagnética incidente e não da sua intensidade. 
O físico Phillip Lenard (1862-1947) estudou experimentalmente o efeito fotoelétrico. Seus experimentos consistiram em justapor paralelamente dentro de um tubo de vácuo duas placas metálicas a uma certa distância uma da outra e ligadas a uma bateria. Para efetuar as medidas, Lenard acoplou ao circuito um amperímetro para medir a corrente elétrica entre as duas placas e um voltímetro para medir a tensão elétrica. Ao iluminar uma das placas com luz de determinadas frequências, fotoelétrons eram ejetados da placa iluminada e alcançavam a outra placa, acelerados pela diferença de potencial fornecida pela bateria e que podia ser registrada pelo voltímetro. A corrente elétrica entre as placas gerada pelos fotoelétrons era medida pelo amperímetro. Lenard notou que ao aumentar a intensidade da luz, mais elétrons eram ejetados a cada segundo. Entretanto, ao manter a frequência da fonte luminosa constante, a energia dos fotoelétrons ejetados não se alterava.

No experimento de Lenard, ao acionar a bateria, a diferença de potencial no circuito possibilitava que mesmo os fotoelétrons ejetados da placa com energia cinética nula chegassem na outra placa. Quando os fotoelétrons ejetados alcançavam a segunda placa, ocorria a saturação da corrente elétrica, ou seja, a corrente se mantinha constante. Logo, quanto maior é a intensidade luminosa maior é a corrente elétrica formada entre as placas. Isso significa que a corrente de saturação depende da intensidade da luz incidente.

Entretanto, quando se inverte a direção do potencial elétrico a fim de retardar o fluxo dos fotoelétrons entre as placas, evidencia-se a existência de um potencial elétrico mínimo, $V_{o}$, denominado potencial de corte, que quanto atingido nenhum fotoelétron alcança a placa de destino. Tal observação indica que a energia cinética inicial com a qual os fotoelétrons são ejetados da placa de origem não depende da intensidade luminosa. A energia cinética máxima é dada pela Equação 4.

$$
K_{\text {max }}=e \cdot V_{o}
$$

onde $e$ é a carga fundamental que vale $1,6 \times 10^{-19} \mathrm{C}$.

Os gráficos na Figura 1 sintetizam as previsões da Equação 2. Vale notar que os dados experimentais disponíveis em 1905 eram pouco precisos para corroborar ou contestar de forma conclusiva a teoria de Einstein. Foram necessários mais onze anos até que, em 1916, Robert Millikan (1868-1953) publicasse os resultados de seus série de experimentos a respeito do efeito fotoelétrico.

Figura 1 - Síntese em gráficos do efeito fotoelétrico, previsto pela equação de Einstein de 1905, Equação 2, e verificado experimentalmente por Lenard entre 1900 e 1902.

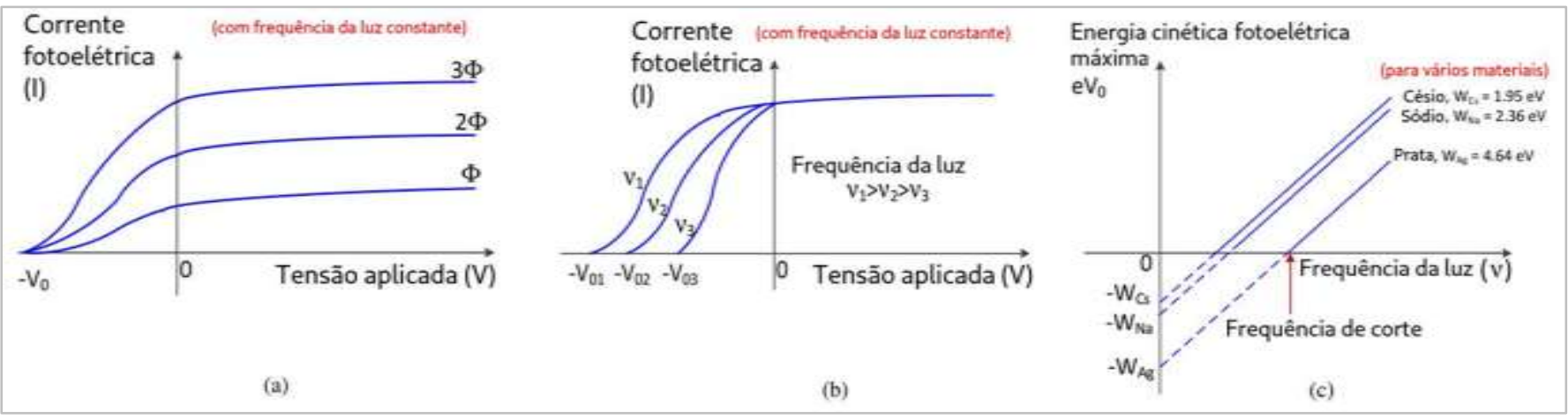

Fonte: Adaptado de Rablau (2019). 
O gráfico da Figura 1(a) relaciona a corrente elétrica produzida pelos fotoelétrons ejetados da placa iluminada e capturados pela outra placa com o potencial elétrico que se estabelece entre elas.

Na Figura 1(b), o gráfico da corrente fotoelétrica versus a voltagem, mostra o comportamento do efeito fotoelétrico ao variar a frequência luminosa. Na Figura 1(c), o gráfico das frequências de corte e a função trabalho para alguns materiais. $\mathrm{Na}$ Tabela 2 apenas a título de ilustração, destacam-se alguns valores adicionais da função trabalho de mais alguns materiais.

Dentre as aplicações tecnológicas baseadas no efeito fotoelétrico, a que interessa abordar neste artigo é célula fotovoltaica, que é a unidade básica dos "painéis solares". É através deles que se dá a captação da radiação solar incidente para iniciar a geração de corrente elétrica. Segundo Duffie, Beckman \& Blair (2020), as células ou conversores fotovoltaicos são dispositivos semicondutores de silício com junção $\mathrm{P}-\mathrm{N}^{1}$ com 20 linhas de grade por centímetro, que convertem parte da radiação solar incidente diretamente em energia elétrica. A seguir este processo será descrito em mais detalhes.

\section{Tipos de Sistemas de Energia Fotovoltaica}

A energia fotovoltaica, como é informalmente conhecida, é a energia elétrica produzida a partir da captação do calor e da luz solar incidente através de um dispositivo denominado "painel solar" usado para captar a energia solar e dar início ao processo de transformação de energia elétrica útil. O painel solar é constituído por unidades denominadas células solares que recebem os fótons da radiação solar incidente e ejetam fotoelétrons como discutido anteriormente.

As fotocélulas são "sanduichadas" entre lâminas de vidro temperado e emolduradas por alumínio como mostrado na Figura 2.

Figura 2 - Partes componentes típicas de um painel solar comercial.

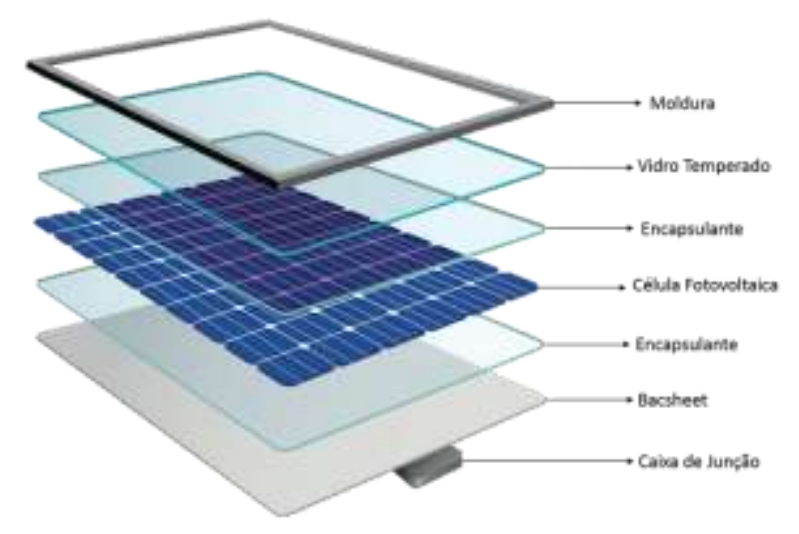

Fonte: Adaptado de The Renewable Energy Hub (2021).

Os painéis solares operam num sistema em conjunto com outros dispositivos que atuam para estabilizar e converter a corrente contínua originalmente gerada nos painéis em corrente alternada que é o padrão compatível na entrada de alimentação da maioria das máquinas e eletrodomésticos². No website The Renewable Energy Hub (2021) tem-se que o sistema é composto

\footnotetext{
${ }^{1}$ Junção ou juntas P-N são produzidas quando dois materiais semicondutores, um do tipo P e outro do tipo N são conectados de modo a manter a continuidade da rede cristalina entre eles. Os dispositivos semicondutores são usados em componentes eletrônicos e possuem junções P-N como sua estrutura básica (Malvino \& Bates, 2016).

${ }^{2}$ A rigor a maior parte dos equipamentos eletroeletrônicos (computadores, TVs, videogames etc.) operam em corrente contínua internamente. $\mathrm{Na}$ entrada de alimentação chega a corrente alternada, mas é retificada e utilizada como corrente contínua. Motores elétricos em geral como os presentes nas geladeiras, batedeiras, liquidificadores etc. usam a corrente alternada (Cruz, 2018).
} 
por painéis solares, um dispositivo inversor (conversor) de corrente contínua para corrente alternada, componentes de proteção (fusíveis, dispositivos de proteção contra surto e disjuntores) além da estrutura para fixação de todo o sistema.

O sistema fotovoltaico on-grid é um sistema conectado à rede da empresa concessionária de energia elétrica. Produz a energia elétrica que alimenta em tempo real os eletrônicos e eletrodomésticos que estão em uso, sendo assim, não consume energia da rede da distribuidora, pois se o sistema produz energia suficiente para atender a demanda. No sistema on-grid a energia excedente produzida é injetada na rede da concessionária e é contabilizada como crédito do usuário que gerou o excedente. Durante a noite, a energia excedente repassada à rede ao longo do dia é então consumida. O sistema on-grid dispensa o uso de baterias, visto que a própria distribuidora é quem faz o armazenamento da energia gerada.

O segundo tipo é o sistema fotovoltaico off-grid. Este tipo de sistema é autônomo pois dispensa o sistema de distribuição da rede elétrica. Como parte do sistema há um banco de baterias projetado para armazenar a energia excedente para consumo em casos de ocorrência de pouca captação de energia, como por exemplo, durante condições meteorológicas desfavoráveis (tempo nublado, chuvas etc.) ou durante à noite. O sistema off-grid é o sistema ideal para quem pretende se desligar da rede de distribuição de energia e se tornar auto suficiente, produzindo sua própria energia o que reduz também o impacto ao meio ambiente (The Renewable Energy Hub, 2021).

Existe ainda o sistema híbrido. Trata-se de um sistema com as capacidades dos dois sistemas anteriores, em termos simples, é um sistema on-grid mas com o banco de baterias do sistema off-grid. Este sistema é recomendado para usuários que não podem ficar sem energia elétrica, tais como hospitais, laboratórios, sistemas de refrigeração de alimentos perecíveis etc. O sistema híbrido trabalha em sincronia com a rede da concessionária e, no instante em que a rede de distribuição sofre algum distúrbio e o abastecimento é suspenso, o off-grid entra em atividade mantendo todo o sistema de fornecimento de energia elétrica do usuário em operação.

Todos estes elementos foram considerados ao projetar o aplicativo descrito a seguir.

\section{O Aplicativo}

A ideia inicial da proposta evoluiu para a criação de um aplicativo para dispositivos móveis baseados no sistema operacional Android, capaz de simular a captação e o consumo de um sistema solar fotovoltaico off-grid, utilizando as especificações técnicas do estado da arte dos equipamentos usados para projetar um sistema residencial com parâmetros realísticos. A opção pelos dispositivos móveis ao invés de qualquer outro, como computadores por exemplo, se deve à ampla disponibilidade de smartphones entre os alunos, a portabilidade e possibilidade de suportar aprendizagem ubiquidade conforme apontam Hightower e Borriello (2001) e Santaella (2013).

O aplicativo apresenta ao usuário uma interface simples e intuitiva, como mostra a Figura 3. 
Figura 3 - Interface do aplicativo que simula o funcionamento de um sistema fotovoltaico autônomo (off-grid).

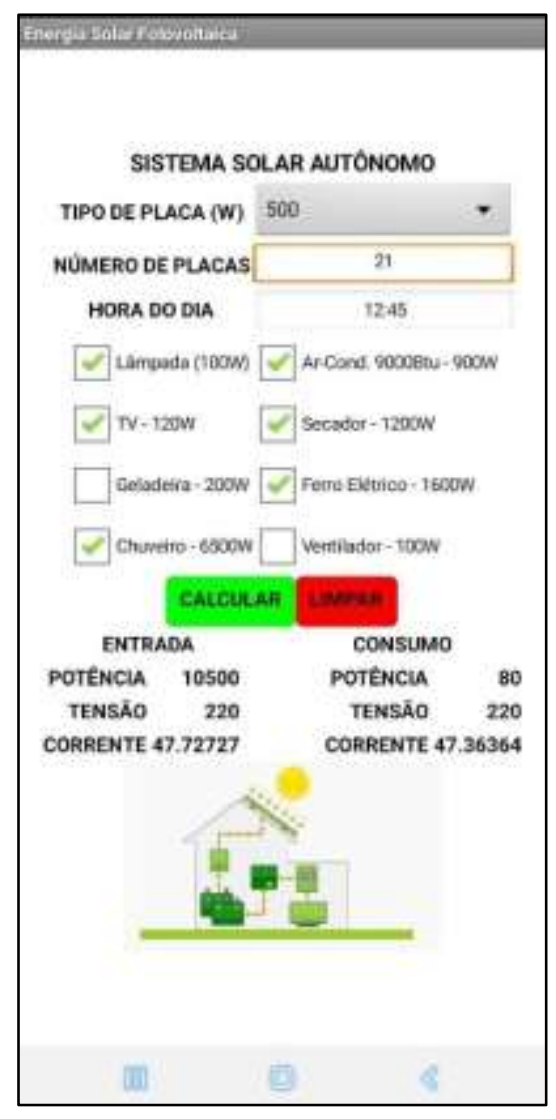

Fonte: Autores.

A interface é constituída por quatro segmentos ou partes distintas, cada uma com um propósito específico e bem definido para total clareza do utilizador.

O primeiro segmento da interface tem três elementos como é mostrado na Figura 4.

Figura 4 - Parâmetros iniciais do sistema fotovoltaico autônomo.

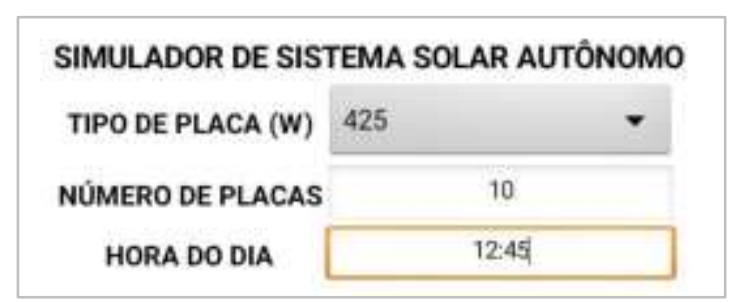

Fonte: Autores.

- $\quad$ TIPO DE PLACA (W) - seletor que oferece ao usuário uma lista com modelos pré-definidos de placas solares comerciais com suas respectivas potências.

- NÚMERO DE PLACAS - campo numérico o qual o usuário deve fornecer a quantidade de placas que comporá o sistema fotovoltaico que será simulado.

- HORA DO DIA - local no qual deverá ser informado hora e minuto do dia cujo rendimento do sistema fotovoltaico será simulado. Como se viu anteriormente, o balanço de energia solar depende das horas de insolação, portanto cada 
época do ano e hora do dia tem um rendimento especifico que envolve de radiação solar incidente e a posição do Sol em relação a placa fotovoltaica.

O segundo segmento da interface com o usuário, apresentado na Figura 5, dispõe alguns eletrodomésticos com suas respectivas potências. Cada eletrodoméstico pode ser selecionado para compor o conjunto de aparelhos que deverão ser alimentados pelo sistema simulado. A seleção dos itens é simples e pode ser efetuada com um toque sobre o aparelho desejado.

Figura 5 - Seletor dos eletrodomésticos no aplicativo.

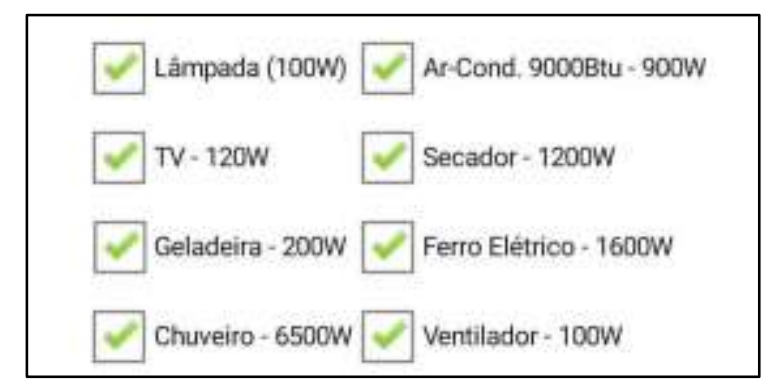

Fonte: Autores.

O terceiro segmento da interface com o usuário, apresentado na Figura 6, tem apenas dois botões clicáveis: o botão CALCULAR e o botão LIMPAR.

Após selecionar e fornecer os parâmetros solicitados no primeiro e segundo segmentos, o botão CALCULAR inicia a simulação com as condições de contorno escolhidas tanto para o sistema captador e transformador de energia solar em energia elétrica, quanto para o conjunto de eletrodomésticos que será alimentado pela eletricidade gerada. O botão LIMPAR zera todas as condições de contorno da simulação e prepara o aplicativo para receber nova configuração a ser simulada.

Figura 6 - Os botões que iniciam a execução da simulação e que reinicia os parâmetros preparando nova simulação.

\section{CALCULAR LIMPAR}

Fonte: Autores.

O quarto segmento da interface com o usuário, apresentado na Figura 7, resume os valores de entrada da energia solar captada e convertida para energia elétrica e os valores de consumo dos eletrodomésticos. A simulação efetua os cálculos baseados em valores de energia gerados e consumidos "em tempo real", ou seja, em função da hora do dia, da capacidade de total de energia armazenada e o tempo máximo que este sistema poderá fornecer energia na ausência de Sol (períodos noturnos ou nublados). 
Figura 7 - Interface do aplicativo com o usuário. Os valores de entrada e de saída da Potência (Watt), Tensão (Volt) e Corrente (Ampére) após cada simulação.

\begin{tabular}{|crrr|}
\hline \multicolumn{2}{c}{ ENTRADA } & \multicolumn{2}{c|}{ CONSUMO } \\
POTÊNCIA & 10500 & POTÊNCIA & 80 \\
TENSÃO & 220 & TENSĀO & 220 \\
CORRENTE & 47.72727 & CORRENTE 47.36364 \\
\hline
\end{tabular}

Fonte: Autores.

Quando uma inconsistência é detectada pelo aplicativo uma mensagem de aviso é apresentada ao usuário que deve intervir conforme o caso. Por exemplo, em caso de subdimensionamento do sistema de captação em relação às necessidades de consumo do conjunto de eletrodomésticos selecionados, a simulação é interrompida. $\mathrm{O}$ aplicativo avisa ao usuário por meio de mensagens de alerta, como mostrado na Figura 8.

Figura 8 - Mensagem de alerta para ocorrências de subdimensionamento.

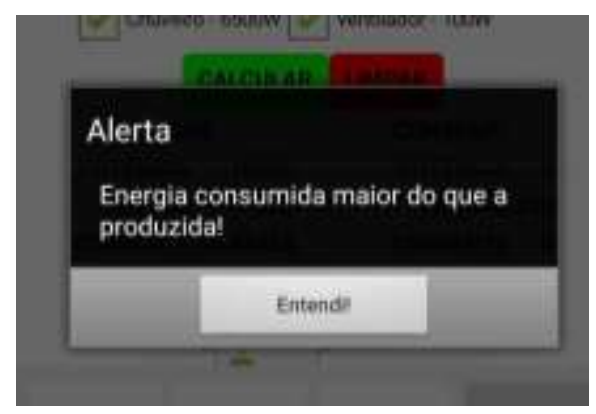

Fonte: Autores.

Este tipo de evento exige a intervenção do usuário no sentido de dar um toque no botão Entendi! e outro toque no botão LIMPAR (ver Figura 6) para reiniciar uma nova execução de simulação corrigindo o problema apontado na mensagem.

\section{Oportunidades para o Ensino, a Aprendizagem e o Debate}

O aplicativo pode ser utilizado durante as sessões presenciais ou remotas de ensino de Física, em conjunto com exposições pelo professor a respeito de conceitos de eletromagnetismo, ondas, termodinâmica e de mecânica quântica. Além disso, há também a sugestão da possibilidade de trabalho interdisciplinar, envolvendo outras disciplinas interessadas em discutir de modo mais amplo o panorama contemporâneo a respeito da matriz energética e da necessidade de desenvolver processos tecnológicos para aproveitamento de fontes de energias renováveis.

O ensino tecnológico também pode beneficiar-se do uso do aplicativo. Neste âmbito, não apenas para apresentar os conteúdos de Física, mas para efetuar simulações que auxiliem no projeto de sistemas off-grid de energia fotovoltaica solar realistas uma vez que os painéis incorporados no aplicativo são de fato os disponíveis no mercado.

\section{Conclusão}

Além de oportunizar e ilustrar a abordagem de temas relacionados com a transformação de energia, ondulatória, conceitos de mecânica quântica, termodinâmica entre outros típicos da Física, a energia fotovoltaica possibilita também discutir em sessões de ensino questões atuais e recorrentes no cotidiano como a da dependência da matriz energética de combustíveis fósseis e da hidroeletricidade, bem como o uso de fontes de energia alternativa, renovável, limpa e sustentável. 
O aplicativo, como recurso didático, viabiliza o aprendizado e o desenvolvimento da intuição de conceitos da Física a respeito de como ocorre a captação, armazenamento e consumo da energia solar fotovoltaica de uma forma intuitiva usando um smartphone que além de portátil é onipresente no cotidiano das pessoas, tornando-o um excelente recurso para a promoção da aprendizagem ubíqua.

$\mathrm{Na}$ continuação desta proposta, pretende-se aperfeiçoar o aplicativo, dotando-o de módulos adicionais contendo aspectos conceituais da Física detalhando e aprofundando aspectos da física solar entre outros que forem identificados pelos usuários como essenciais ao entendimento e à descrição do aproveitamento da energia solar para a produção de energia elétrica pelo fenômeno fotovoltaico, tornando-o cada vez mais um "repositório" de conhecimento a respeito do assunto, portátil e autocontido num aplicativo para dispositivos móveis tais como os tablets e os smartphones.

Nas versões futuras do aplicativo pretende-se incluir também a possibilidade de simular sistemas on-grid e híbridos. A inclusão de animações que permitam visualizar de forma dinâmica o funcionamento dos processos durante cada execução é também uma possibilidade em estudo. Os autores pretendem disponibilizar o aplicativo via Google Play Store assim que alguns detalhes estéticos da interface com o usuário forem redesenhados.

\section{Agradecimentos}

Agradecemos aos avaliadores pelas sugestões pertinentes e objetivas que contribuíram para aprimorar a qualidade deste artigo.

\section{Referências}

Corrêa, N. B.O. de, \& Benite, C. R. M. (2020). Fontes Renováveis de Energia: uma Abordagem Interdisciplinar no Ensino de Física. Editora Appris.

Cruz, E. C. A. (2018). Circuitos elétricos: corrente contínua e corrente alternada. Érica/Saraiva Educação.

Duffie, J. A., Beckman W. A. \& Blair, N. (2020). Solar Engineering of Thermal Processes, Photovoltaics and Wind. New York, John Wiley \& Sons, (5a ed.).

Griffiths D. J. (2017). Introduction to Quantum Mechanics. United Kingdom: Cambridge University Press.

Hightower, J., \& Borriello, G. (2001). Location systems for ubiquitous computing. Computer, 34(8), 57-66.

Horton, R. et al. (1996). Crop residue effects on surface radiation and energy balance. Theoretical and Applied Climatology, 54(1), $27-37$.

Kejna, M., Uscka-Kowalkowska, J., \& Kejna, P. (2021). The influence of cloudiness and atmospheric circulation on radiation balance and its components. Theoretical and Applied Climatology, 144(3), 823-838.

Lima, C. E. (2018). A energia fotovoltaica num contexto CTSA: Uma sequência de ensino sobre as transformações de energia solar em energia elétrica. 2018. Dissertação (Mestrado Profissional Educação e Docência) - Universidade Federal de Minas Gerais, Belo Horizonte.

Lu, Y. et al. (2021). Study on Solar Radiation and the Extreme Thermal Effect on Concrete Box Girder Bridges. Applied Sciences, 11(14), 6332.

Maimoni, F. P., \& Cardoso, R. B. (2020). Análises de viabilidades econômicas para alternativas de utilização da energia solar em residência do Estado de Minas Gerais, Brasil. Research, Society and Development, 9(8), e853986221. https://doi.org/10.33448/rsd-v9i8.6221

Malvino, A. \& Bates, D. (2016). Eletrônica. (8a ed.), AMGH, v.1

Nasa. National Aeronautics and Space Administration. New NASA Data Sheds (Sun) Light on Climate Models. https://www.nasa.gov/feature/goddard/2021/newnasa-data-sheds-sun-light-on-climate-models.

Rablau, C. et al. (2019). The photoelectric effect-project-based undergraduate teaching and learning optics through a modern physics experiment redesign. In: Education and Training in Optics and Photonics. Optical Society of America, 11143.

Rocha, F. L. M., \& Costa, F. E. M. (2019). Energia solar fotovoltaica como temática facilitadora da aprendizagem de física quântica na educação básica. Educação \& Linguagem, 6(3), 110-120.

Santaella, L. (2013). Comunicação ubíqua: repercussões na cultura e na educação. Paulus (Coleção comunicação).

Silva, C. G. da. (2010) De Sol a Sol: a energia no século XXI. Oficina de Textos.

Silva, J. A. da et al. (2017) Energia solar fotovoltaica: Um tema gerador para o aprendizado de Física. Scientia Plena. 13(1). 
Research, Society and Development, v. 10, n. 15, e279101522946, 2021

(CC BY 4.0) | ISSN 2525-3409 | DOI: http://dx.doi.org/10.33448/rsd-v10i15.22946

Sobczyk, W. et al. (2017). Ecological and economical aspects of solar energy use. In E3S Web of Conferences (Vol. 14, p. 01011). EDP Sciences.

The Renewable Energy Hub. (2021) A Complete guide to solar panels in 2020. Recuperado de https://www.renewableenergyhub.co.uk/main/solar-panels/.

Von Schuckmann, K et al. (2020). Heat stored in the Earth system: where does the energy go? Earth System Science Data, 12(3), 2013-2041.

Wendisch, M., \& Yang, P. (2012). Theory of atmospheric radiative transfer: a comprehensive introduction. John Wiley \& Sons.

Workfunction Values (Reference Table). (2020). https://chem.libretexts.org/@go/page/41952. 